\title{
Ressecção pulmonar após transplante cardíaco ortotópico
}

\author{
Noedir A. G. STOLF*, Alfredo I. FIORELLI ${ }^{*}$, Fernando BACAL*, Viviane VEIGA*, \\ Ricardo BERNADIS* ${ }^{*}$ Anderson BENÍCIO*, Edimar A. BOCCHI ${ }^{*}$, Moise DALVA*, Patrícia M. CURY*
}

RBCCV 44205-488

Stolf N A G, Fiorelli A I, Bacal F, Veiga V, Bernadis R, Benício A, Bocchi E A, Dalva M, Cury P M - Ressecção pulmonar após transplante cardíaco ortotópico. Rev Bras Cir Cardiovasc 2000; 15 (1): 66-71.

RESUMO: Objetivos: O objetivo do presente estudo é apresentar e discutir a evolução clínica de 2 pacientes que, no pós-operatório de transplante cardíaco ortotópico, apresentaram formação de abscesso pulmonar em conseqüência de infecção pulmonar complicada.

Casuística e Métodos: Em ambos os casos, a lobectomia foi indicada como último recurso em virtude da falta de resposta ao tratamento clínico convencional. No ato operatório, houve rigor na observância dos preceitos clássicos adotados, habitualmente, nas ressecções pulmonares para se evitar o aparecimento de fístulas broncopleurais.

Resultados: Os pacientes apresentaram boa evolução pós-operatória e resolução definitiva do quadro infeccioso e, atualmente, encontram-se em seguimento pós-operatório em condições clínicas estáveis.

DESCRITORES: Transplante do coração, efeito adverso. Abscesso pulmonar, cirurgia. Pneumonectomia. Rejeição de enxerto, etiologia.

\section{INTRODUÇÃO}

A imunossupressão, apesar de controlar a rejeição após o transplante de órgãos, facilita o aparecimento de infecções no pós-operatório. No transplante cardíaco, o trato respiratório é um dos sítios mais freqüentemente acometidos pelas bactérias e vírus. Os principais fatores que predispõem às infecções pulmonares são congestão pulmonar secundária à insuficiência cardíaca de longa evolução, doenças pulmonares preexistentes, instabilidade hemodinâmica pós-operatória e disfunção de outros órgãos (1).

$\mathrm{Na}$ seleção dos candidatos ao transplante cardíaco tem-se dedicado especial atenção na avaliação do comportamento da função pulmonar, procurando-se identificar a existência de potenciais fatores de risco pós-operatório. Deve-se ressaltar que a hipertensão pulmonar, a doença pulmonar crônica obstrutiva avançada, a infecção pulmonar em atividade e a embolia pulmonar seguida de infarto podem ser considerados critérios de exclusão temporária ou definitiva da lista de espera, em virtude da maior incidência de disfunção do enxerto ou infecção ${ }^{(2)}$.

Os pacientes selecionados para o transplante cardíaco, que apresentam risco adicional de tromboembolismo à doença cardíaca ou já apresentaram eventos confirmados, devem receber anticoagulação profilática até o momento da operação $(3,4)$.

Após o transplante cardíaco, as complicações tromboembólicas, incluindo tromboflebite, morte súbita, infarto agudo do miocárdio ou pulmonar, diminuem significativamente; contudo, mantêm-se elevadas em relação à população em geral. FORRAT et al. (4), em 1996, encontraram 9,8\% destas complicações após o transplante cardíaco, das quais $3,9 \%$ foram fatais.

Trabalho realizado no Instituto do Coração do Hospital das Clínicas da Faculdade de Medicina da Universidade de São Paulo. São Paulo, SP, Brasil. Recebido para publicação em setembro de 1999.

* Do Instituto do Coração do Hospital das Clínicas da Faculdade de Medicina da Universidade de São Paulo.

Endereço para correspondência: Noedir A.G. Stolf. Rua João Lourenço, 386. São Paulo, SP, Brasil. CEP 04508-030. Tel. (011) $3069-5318$. e-mail: stolf@incor.usp.br 
Stolf N A G, Fiorelli A I, Bacal F, Veiga V, Bernadis R, Benício A, Bocchi E A, Dalva M, Cury P M - Ressecção pulmonar após transplante cardíaco ortotópico. Rev Bras Cir Cardiovasc 2000; 15 (1): 66-71.

A embolia ou infarto pulmonar são fatores de exclusão temporária dos candidatos em lista de espera, devido ao maior risco de aparecimento de infecção e abscesso pulmonar no pós-operatório ${ }^{(3)}$. A antibioticoterapia específica, a drenagem brônquica e a manutenção de fisioterapia respiratória adequada representam os pilares de sustentação do tratamento clínico desta grave complicação. As ressecções pulmonares constituem-se no último recurso e somente estão indicadas nos casos de falha de resposta à antibioticoterapia aplicada, muito embora apresentem risco adicional de fístulas brônquicas devido ao uso crônico de corticóide ${ }^{(5-7)}$. Diante deste último fato, diferentes opções operatórias têm sido propostas no tratamento cirúrgico do coto brônquico durante as ressecções pulmonares, visando reduzir o risco destas fístulas (8).

O presente estudo tem por objetivo relatar e discutir a evolução clínica de 2 pacientes submetidos ao transplante cardíaco ortotópico que, no pósoperatório, desenvolveram abscesso pulmonar em decorrência de infecção pulmonar complicada e necessitaram de lobectomia para erradicação do foco infeccioso.

\section{RELATO DOS CASOS}

\section{Caso $N^{\circ} 1$}

Trata-se de paciente de 66 anos, do sexo feminino, portadora de insuficiência cardíaca congestiva devido a cardiomiopatia dilatada de longa evolução. O transplante cardíaco foi indicado por falta de resposta ao tratamento clínico convencional. A paciente não apresentava antecedentes de tromboembolismo e tabagismo. Referia apenas asma brônquica controlada há 33 anos e a espirometria revelou presença de distúrbio ventilatório obstrutivo de pequena magnitude. Na radiografia de tórax observava-se imagem de importante cardiomegalia, sinais de congestão pulmonar com aumento da trama vasobrônquica.

A paciente foi submetida ao transplante cardíaco ortotópico e apresentou boa evolução clínica imediata, recebendo alta hospitalar com esquema tríplice de imunossupressão, ciclosporina, azatioprina e corticóide. Os episódios de rejeição foram acompanhados com biópsia endomiocárdica e cintilografia miocárdica com Gálio-67.

Dois meses após o transplante, a paciente apresentou esofagite por vírus Herpes simplex, identificado pela endoscopia digestiva, que regrediu com tratamento medicamentoso. Vinte dias após este evento, foi internada com quadro de dor abdominal de forte intensidade em hipocôndrio direito, icterícia e febre. A suspeita clínica de colecistite aguda foi confirmada pelos exames laboratoriais e pela ultrasonografia. O tratamento cirúrgico foi indicado e consistiu em colecistectomia e biópsia hepática, onde foram encontrados múltiplos microcálculos no interior da vesícula biliar e não houve crescimento de qualquer agente patogênico.

Um mês após este último evento, a paciente retornou ao hospital com piora do estado geral, dor torácica, febre, tosse e expectoração purulenta. $\mathrm{Na}$ radiografia de tórax observou-se a presença de opacidade arredondada no terço médio do pulmão direito, que evoluiu progressivamente para cavitação (Figura 1). A tomografia computadorizada de tórax de alta resolução confirmou a referida imagem, porém, agora com nível líquido no seu interior, projetando-se na região periférica do lobo superior direito. Estabelecido o diagnóstico de abscesso pulmonar, iniciou-se a antibioticoterapia com clindamicina e, posteriormente, ciprofloxacina, associada a intensa fisioterapia respiratória. A broncofibroscopia foi realizada também na tentativa de drenagem por via endoscópica, porém sem sucesso. Como último recurso, indicou-se a ressecção pulmonar, pela dificuldade de controle da infecção ao tratamento clínico proposto.

Durante $o$ ato operatório, notou-se que o abscesso acometia grande parte do lobo superior direito e que as demais áreas do parênquima pulmonar encontravam-se retraídas e endurecidas. Optou-se pela lobectomia, que foi realizada de forma convencional e o coto brônquico foi fechado com pontos separados em "8" com fio monofilamentar de polipropileno $4-0$, sem qualquer tipo de revestimento para proteção. A cultura do material retirado do interior do abscesso revelou crescimento de Klebsiella pneumoniae. A paciente apresentou resolução definitiva do processo infeccioso e, atualmente, encontra-se em condições clínicas estáveis no $69^{\circ}$ mês de seguimento pós-operatório da lobectomia e no $73^{\circ}$ mês do transplante cardíaco (Figura 2).

\section{Caso $N^{\circ} 2$}

Trata-se de paciente de 66 anos, do sexo masculino, portador de cardiomiopatia dilatada de etiologia isquêmica, com relativa caquexia cardíaca e insuficiência renal crônica. O transplante cardíaco foi indicado em virtude da insuficiência cardíaca mostrar-se refratária ao tratamento clínico convencional. A avaliação da função pulmonar do paciente evidenciou presença de doença pulmonar obstrutiva crônica discreta, sem sinais recentes de embolia ou infarto pulmonar. Na radiografia de tórax evidenciavam-se sinais de congestão pulmonar e cardiomegalia. O transplante cardíaco 
Stolf N A G, Fiorelli A I, Bacal F, Veiga V, Bernadis R, Benício A, Bocchi E A, Dalva M, Cury P M - Ressecção pulmonar após transplante cardíaco ortotópico. Rev Bras Cir Cardiovasc 2000; 15 (1): 66-71.

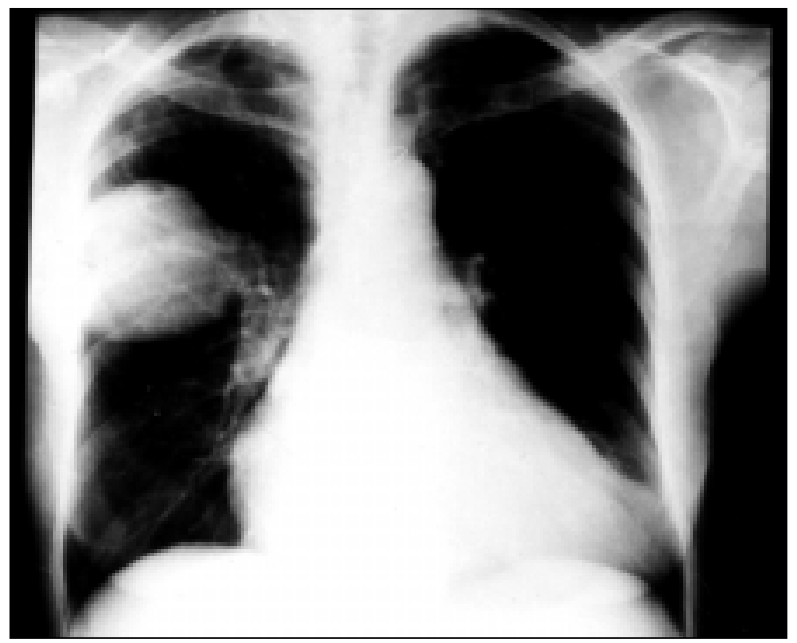

Fig. 1 A -Radiografia de tórax em posição póstero-anterior, com presença de opacidade arredondada no lobo superior direito.

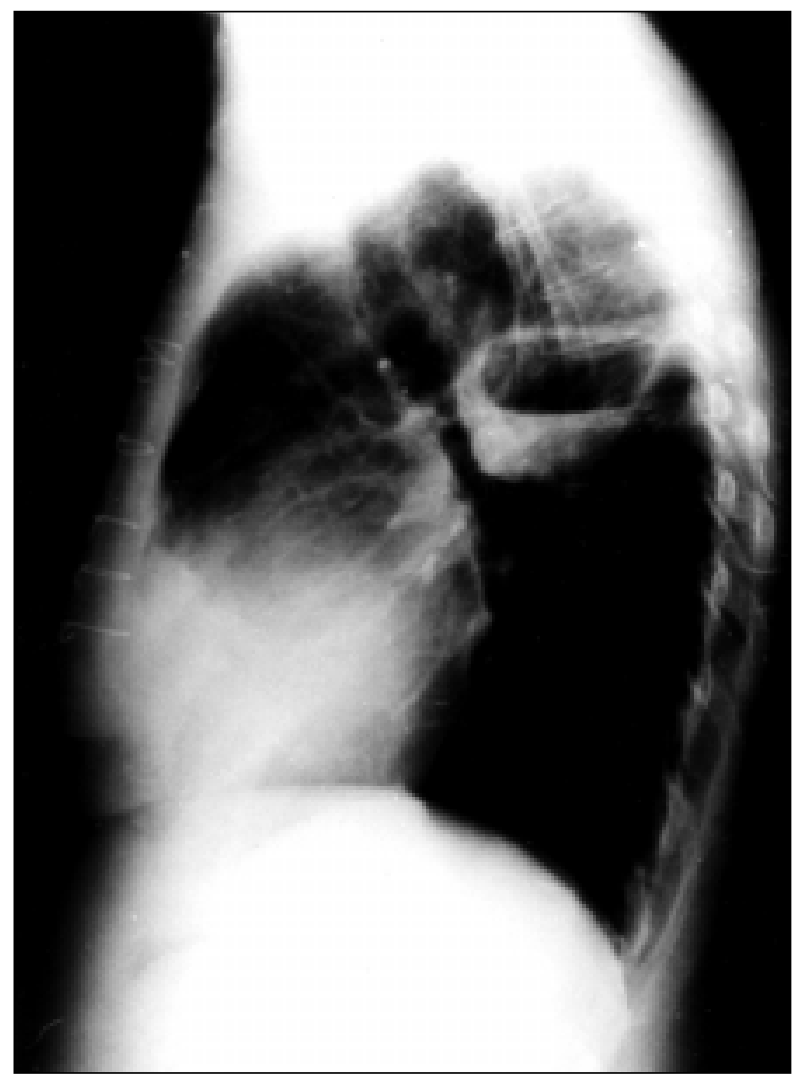

Fig. 1 B -Perfil esquerdo- Evolução para abscesso com nível líquido no seu interior.

ortotópico foi realizado em caráter de urgência devido a progressão do quadro clínico para choque cardiogênico severo, que se manteve por vários dias, com necessidade de administração de altas doses de fármacos vasoativos. Não houve qual- quer intercorrência durante 0 ato operatório e o paciente apresentou boa recuperação clínica.

No pós-operatório imediato, apresentou rejeição aguda sem repercussão hemodinâmica, diagnosticada por biópsia endomiocárdica e tratada com pulsoterapia endovenosa de corticóide. Um mês após o transplante, o paciente foi submetido ao implante de marcapasso definitivo, devido a persistência de bloqueio atrioventricular total intermitente. Nesse período, apresentou diminuição dos murmúrios vesiculares no terço inferior do hemitórax direito. A radiografia, a ultra-sonografia e a tomografia computadorizada de tórax (Figura 3) confirmaram a presença de derrame pleural à direita e atelectasia do lobo inferior.

Houve piora da insuficiência renal crônica no segundo mês de evolução pós-operatória, acompanhada de acidose metabólica e hiperpotassemia. Ao exame físico, o paciente apresentava-se com taquipnéia, presença de estertores crepitantes e subcrepitantes na metade inferior do hemitórax direito. $\mathrm{Na}$ tomografia computadorizada de tórax podia observar-se a presença de imagem de opacificação heterogênea, em lobo inferior do pulmão direito, sugestiva de abscesso pulmonar.

A cultura do material retirado do interior do abscesso por broncofibroscopia revelou crescimento de Pseudomonas aeruginosa sensível à ceftazidima. A falta de resposta ao tratamento clínico motivou a indicação da ressecção cirúrgica. Durante 0 ato operatório, observou-se que o abscesso comprometia grande parte do lobo inferior e o restante do parênquima pulmonar encontrava-se enrijecido com atelectasia. Frente a este aspecto macroscópico, a alternativa de ressecção parcial mostrou-se inválida e optou-se pela lobectomia inferior direita. A ressecção pulmonar adotada foi realizada de forma

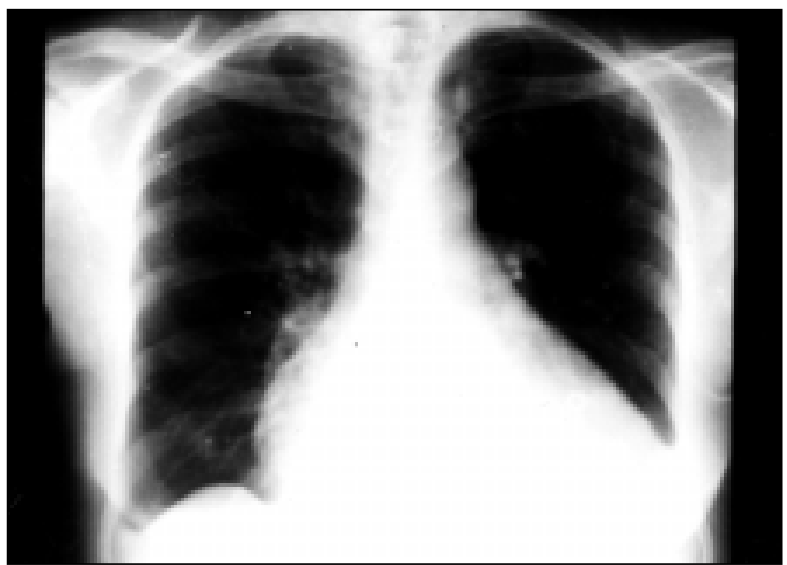

Fig. 2 - Radiografia de tórax de controle, em incidência póstero-anterior, realizada 69 meses após lobectomia superior direita. 


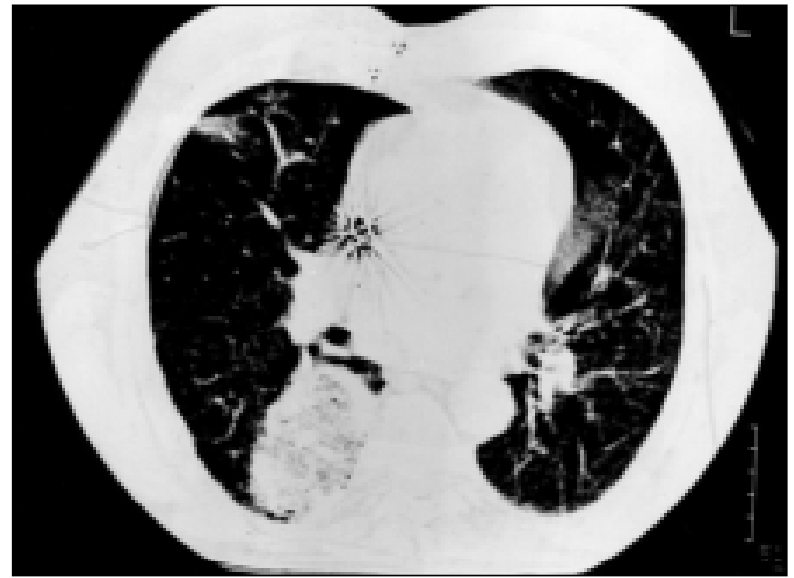

Fig. 3 - Tomografia computadorizada de tórax com imagem de abscesso pulmonar identificado pela opacidade heterogênea localizada em lobo inferior direito.

convencional e o coto brônquico foi fechado com pontos separados em "8" com fio monofilamentar de polipropileno 4-0 e protegido com segmento de pleura mediastinal.

Dois meses após a lobectomia, o paciente apresentou novo derrame pleural à direita, seguido de empiema, que melhorou após drenagem torácica e antibioticoterapia específica. A cultura do líquido pleural revelou crescimento de Staphylococus aureus sensível à vancomicina. Atualmente, o paciente encontra-se bem no $6^{\circ}$ mês de seguimento pósoperatório da lobectomia e no $9^{\circ}$ mês de transplante cardíaco.

\section{COMENTÁRIOS}

A despeito dos avanços técnicos no transplante cardíaco, as infecções em muitas séries constituem a principal causa de óbito imediato e do tardio. Os episódios de infecção são mais freqüentes nos primeiros meses após o transplante, quando a imunossupressão é mais intensa, ocorrendo, posteriormente, declínio progressivo. O trato respiratório e renal são os sítios mais freqüentemente acometidos pelas bactérias e vírus (1). As infecções pulmonares acometem cerca de 40 a $70 \%$ dos pacientes submetidos ao transplante cardíaco e são responsáveis por mais de $50 \%$ dos casos de morte durante o primeiro ano de pós-operatório. Os principais fatores predisponentes às infecções pulmonares são: imunossupressão, disfunção em outros órgãos, intubação prolongada, estado nutricional, doenças ou infecções associadas, tromboembolismo pulmonar, agressão cirúrgica e o desenvolvimento de instabilidade hemodinâmica no pós-operatório imediato $(1,9,10)$.
A imunossupressão própria dos transplantes é o principal fator responsável pela diminuição da imunidade celular e predispõe às infecções causadas por diversos patógenos oportunistas, entre eles destacam-se as bactérias intracelulares (Listeria, Mycobacterium, Salmonella), fungos (Candida, Aspergillus), herpes e os protozoários (Pneumocystis carinii e Toxoplasma gondii) $(1,9)$.

O comportamento das infecções em pacientes imunossuprimidos geralmente é atípico e exige todo esforço na precocidade e segurança do diagnóstico para instituição de terapêutica adequada.

O tipo e a virulência do agente etiológico da infecção desempenham papel fundamental na evolução clínica do paciente. Assim, agentes altamente virulentos com grande capacidade necrosante têm suas ações potencializadas no hospedeiro imunodeprimido. A pneumonia é a infecção que mais freqüentemente acomete os pacientes após o transplante cardíaco e é responsável por cerca de 20 a $40 \%$ dos casos de óbito $(1,9,10)$. O trato respiratório torna-se alvo fácil de infecções devido à intubação orotraqueal prolongada, à agressão da toracotomia, à imunossupressão e a própria insuficiência cardíaca congestiva crônica. O Staphylococcus aureus e as bactérias anaeróbias são os principais agentes etiológicos responsáveis por estas infecções (9).

As infecções pulmonares que evoluem com destruição central do parênquima podem culminar com a formação de abscessos, muitas vezes de difícil resolução clínica e necessitam de ressecção cirúrgica. Os principais agentes responsáveis pela formação dos abscessos pulmonares são Klebsiella pneumoniae e Staphylococcus aureus. Todavia, nos pacientes imunossuprimidos deve-se considerar a participação dos fungos na formação destes abscessos, descritos também como os agentes presentes no pós-operatório de outros transplantes de órgãos $(5,11,12)$.

Durante a seleção dos candidatos ao transplante cardíaco é de fundamental importância a avaliação pulmonar criteriosa e a identificação de fatores de risco. Os pacientes que apresentam resistência vascular pulmonar acima de 6 Unidades Wood, mesmo após a infusão de vasodilatadores, são contra-indicados para o transplante cardíaco ortotópico (2), já que a hipertensão pulmonar aumenta a incidência de falência aguda do enxerto após o transplante e, conseqüentemente, da mortalidade imediata. A doença pulmonar crônica obstrutiva em fase avançada e com severa restrição da capacidade ventilatória também é critério de exclusão definitiva do programa de transplante (2-4).

Por outro lado, a infecção ativa exclui os pacientes temporariamente da lista de espera até que o processo infeccioso se resolva. Esta mesma atitude 
Stolf N A G, Fiorelli A I, Bacal F, Veiga V, Bernadis R, Benício A, Bocchi E A, Dalva M, Cury P M - Ressecção pulmonar após transplante cardíaco ortotópico. Rev Bras Cir Cardiovasc 2000; 15 (1): 66-71.

é adotada na embolia pulmonar aguda, principalmente quando seguida de infarto, o que afasta os pacientes do transplante, por período variável entre três e seis meses. Esta orientação se apóia no risco potencial de infecção pulmonar no pós-operatório, complicada pela formação de abscesso, empiema, fístula broncopleural ou sepse $(1-4,9)$.

Os fenômenos tromboembólicos pulmonares incidem em cerca de 30 a $50 \%$ dos pacientes com cardiomiopatia dilatada candidatos ao transplante $(4,13)$. Esta alta incidência e os riscos de infecções respiratórias após o transplante têm feito com que alguns autores indiquem mais precocemente as ressecções pulmonares, mesmo considerando-se a gravidade da cardiomiopatia $(8,14,15)$. LORIA et al. ${ }^{(14)}$, em 1991, descreveram o emprego de lobectomia pulmonar após infarto pulmonar previamente ao transplante, acreditando que esta tática possa abreviar o retorno dos pacientes à lista de espera e reduzir também os riscos de infecções pulmonares no pós-operatório. Desde que o paciente apresente reserva funcional pulmonar adequada, tem-se descrito a realização de transplante cardíaco em pacientes submetidos previamente a ressecções pulmonares mais extensas $(6,16)$.

Nos casos ora relatados, os pacientes submetidos ao transplante cardíaco ortotópico não apresentavam qualquer evidência de embolia pulmonar como fator de risco na formação dos abscessos. A terapia imunossupressora adotada foi com esquema tríplice convencional e o controle de rejeição com biópsias endomiocárdicas e cintilografia miocárdica com Gálio-67. Contudo, a infecção do trato gastrointestinal, seguida da colecistectomia, possa ter potencializado de forma variável a queda da imunidade, favorecendo a infecção pulmonar, no primeiro caso. No segundo paciente, o estado de choque prolongado no pré-operatório, a caquexia e o derrame pleural com atelectasia seguramente desempenharam papel expressivo na formação do abscesso pulmonar.

O tratamento convencional do abscesso pulmonar envolve a administração de antibióticos, tentativa de drenagem endoscópica, drenagem de empiema (quando presente), além de fisioterapia respiratória adequada. Os abscessos causados por bactérias anaeróbias costumam responder bem ao tratamento com penicilina, clindamicina ou penicilina associada a metronidazol e a lobectomia tem sido reservada como último recurso aos casos refratários ao tratamento clínico ${ }^{(1,9)}$. Somente nas infecções fúngicas têm-se preconizado terapêutica mais agressiva e ressecções pulmonares mais precoces, considerando-se a alta mortalidade causada por estes agentes $(5,11)$.

As ressecções pulmonares após transplante são pouco freqüentes, muito embora exista preocupação constante a respeito do risco aumentado de fístulas brônquicas em virtude da imunossupressão, com especial atenção ao corticóide (6). DIETHRICH et al. (15), em 1987, descreveram o emprego de lobectomia com sucesso após transplante cardiopulmonar por atelectasia persistente, não mencionando qualquer tratamento diferenciado no fechamento do brônquio. Diferentes táticas operatórias na proteção do coto brônquico têm sido preconizadas, enfatizado-se o envolvimento com pericárdio ou epíploon. CAVAROCCHI et al. (8), em 1989, descreveram a realização de lobectomia pulmonar em 2 pacientes no pós-operatório de transplante empregando a rotação de retalho muscular para proteção do coto brônquico e preenchimento do espaço interpleural.

$\mathrm{Na}$ presente série, procurou-se empregar dissecções econômicas do hilo pulmonar para preservar a boa vitalidade do coto brônquico e optou-se pelo fechamento convencional, sem a adoção de medidas mais agressivas.

Deve-se ressaltar que esta mesma atenção deve ser aplicada na elucidação diagnóstica de nódulos pulmonares que aparecem após o transplante, mesmo em pacientes assintomáticos. A precocidade na indicação da ressecção destes nódulos, acompanhadas ou não de segmentos pulmonares, é de fundamental importância na diferenciação diagnóstica entre infecção ou neoplasia, para instituição de terapêutica adequada $(5,17)$.

Concluindo, os pacientes submetidos ao transplante cardíaco apresentam fatores de risco adicional à formação de abscessos pulmonares, e na falta de resposta ao tratamento convencional, deve-se considerar precocemente a ressecção pulmonar como recurso alternativo. 
Stolf N A G, Fiorelli A I, Bacal F, Veiga V, Bernadis R, Benício A, Bocchi E A, Dalva M, Cury P M - Ressecção pulmonar após transplante cardíaco ortotópico. Rev Bras Cir Cardiovasc 2000; 15 (1): 66-71.

RBCCV 44205-488

Stolf N A G, Fiorelli A I, Bacal F, Veiga V, Bernadis R, Benício A, Bocchi E A, Dalva M, Cury P M - Pulmonary resection after orthotopic heart transplantation. Rev Bras Cir Cardiovasc 2000; 15 (1): 66-71.

ABSTRACT: Objective: The purpose of this study is to present and discuss the clinical evolution of 2 patients who after orthotopic heart transplantation presented complicated pulmonary infection, resulting in the formation of abscess.

Material and Methods: In both cases lobectomy was indicated due to failure to respond to conventional clinical treatment. At operation classical rules of pulmonary resection were strictly adhered to avoid the appearance of bronchopleural fistulas.

Results: The patients presented a good postoperative course with definitive resolution of infections and at follow-up are in stable condition.

DESCRIPTORS: Heart transplantation, effect adverse. Lung abscess, surgery. Pneumonectomy. Graft rejection, etiology.

\section{REFERÊNCIAS BIBLIOGRÁFICAS}

1 De Maria R, Minoli L, Parolini M et al. - Prognostic determinants of six-month morbidity and mortality in heart transplant recipients. The Italian Study Group on Infection in Heart Transplantation. J Heart Lung Transplant 1996; 15: 124-35.

2 Naeije R, Lipski A, Abramowicz $M$ et al. - Nature of pulmonary hypertension in congestive heart failure: effects of cardiac transplantation. Am J Respir Crit Care Med 1994; 149 (4 Pt 1): 881-7.

3 Young J N, Yazbeck J, Esposito G, Mankad P, Townsend $E$, Yacoub $M$ - The influence of acute preoperative pulmonary infarction on the results of heart transplantation. J Heart Transplant 1986; 5: 20-2.

4 Forrat R, Ferrera R, Boissonnat $\mathrm{P}$ et al. - High prevalence of thromboembolic complications in heart transplant recipients. Transplantation 1996; 61: 757-62.

5 Baron O, Guillaume B, Moreau P et al. - Aggressive surgical management in localized pulmonary mycotic and nonmycotic infections for neutropenic patients with acute leukemia: report of eighteen cases. $J$ Thorac Cardiovasc Surg 1998; 115: 63-9.

6 Domenegati E, Ciccone R, Reali F, Reposi C, De Amici $D$, Rissotti M - Anesthesiologic management for lower pulmonary lobectomy intervention in patients with heart transplant: a clinical case. Minerva Anestesiol 1989; 55: 477-80.

7 Yonan N A, Egan J, Deiraniya A K, Rahman A N - Right lower lobe herniation after domino heart-lung transplantation. Ann Thorac Surg 1995; 59: 1223-6.

8 Cavarocchi N C, Carp N Z, Mitra A et al. - Successful heart transplantation in recipients with recent preoperative pulmonary emboli. $J$ Heart Transplant 1989; 8: 494-8.
9 Hsu J, Griffith B P, Dowling R D et al. - Infections in mortally ill cardiac transplant recipients. J Thorac Cardiovasc Surg 1989; 98: 506-9.

10 The registry of the International Society for Heart and Lung Transplantation: ninth official-report. $J$ Heart Lung Transplant 1992; 11 (4 Pt 1): 599-606.

11 Gribetz A R, Chuang M T, Burrows L, Teirstein A S Rhizopus lung abscess in renal transplant patient successfully treated by lobectomy. Chest 1980; 77 : 102-4.

12 Lupinetti F M, Behrendt D M, Giller R H, Trigg M E, de Alarcon $\mathrm{P}$ - Pulmonary resection for fungal infection in children undergoing bone marrow transplantation. J Thorac Cardiovasc Surg 1992; 104: 684-7.

13 Stevenson L W \& Perloff J K - The dilated cardiomyopathies: clinical aspects. Cardiol Clin 1988; 6: 187-218.

14 Loria K M, Salinger M H, Frohlich T G, Arentzen C E, Alexander J C, Anderson R W - Right lower lobectomy for pulmonary infarction before orthotopic heart transplantation. J Heart Lung Transplant 1991; 10: 325-8.

15 Diethrich E B, Bahadir I, Gordon M et al. - Postoperative complications necessitating right lower lobectomy in a heart-lung transplant recipient with previous sternotomy. J Thorac Cardiovasc Surg 1987; 94: 389-92.

16 Goenen M, Baele P, Lintermans J et al. - Orthotopic heart transplantation eleven years after left pneumonectomy. J Heart Transplant 1988; 7: 309-11.

17 End A, Helbich T, Wisser W, Dekan G, Klepetko W The pulmonary nodule after lung transplantation: cause and outcome. Chest 1995; 107: 1317-22. 